\title{
CONTEXT DEPENDENT-DYNAMIC PROCESSES IN ATTENTION \\ DEFICIT/HYPERACTIVITY DISORDER: DIFFERENTIATING COMMON \\ AND UNIQUE EFFECTS OF STATE REGULATION DEFICITS AND DELAY \\ AVERSION
}

Edmund J S Sonuga-Barke ${ }^{1,2^{*}}$, Jan R Wiersema ${ }^{2}$, Jacob J van der Meere ${ }^{2,3}$, and Herbert Roeyers ${ }^{2}$.

1. Institute for Disorders of Impulse and Attention, School of Psychology, University of Southampton, UK.

2. Department of Clinical Experimental \& Health Psychology, Ghent University, Belgium.

3. Department of Neuropsychology, University of Groningen, The Netherlands.

${ }^{*}$ Corresponding author 


\section{DISCLOSURES:}

Edmund J S Sonuga-Barke:

Recent speaker board: Shire Pharma, UCB Pharma

Current \& recent consultancy: UCB Pharma, Shire

Current \& recent research support: Janssen Cilag, Shire, Qbtech

Advisory Board: Shire, Flynn Pharma, UCB Pharma, Astra Zeneca

Conference support: Shire

Jan R Wiersema: no disclosures or conflicts of interest to report

Jacob J van der Meere: no disclosures or conflicts of interest to report

Herbert Roeyers: no disclosures or conflicts of interest to report

ACKNOWLEDGMENTS: N.A. 


\section{ABSTRACT}

The ability to specify differential predictions is a mark of a scientific models' value. State regulation deficits (SRD) and delay aversion (DAv) have both been hypothesized as context-dependent dynamic dysfunctions in ADHD. However, to date there has been no systematic comparison of their common and unique elements. Here we review these hypotheses - and describe the core and secondary manifestations of the two constructs and review evidence In support of them. Second, we focus on what are seen as the hallmark indicators of the two deficits - preference of small immediate over large delayed rewards for DAv and the slow event rate effect for SRD. We describe the overlap between these two manifestations and then explore how experimental manipulations and the analysis of neuropsychological and physiological mediators of effects can allow us to differentiate these two patterns of neuropsychological dysfunction on the basis of specific predictions. Finally, we highlight the implications of neuropsychological heterogeneity for the practical implementation of tests of DAv and SRD.

Key words: Attention Deficit/Hyperactivity Disorder; delay aversion; state regulation; cognitive energetic; event rate; delay of gratification; delayed reward. 


\section{MOVING BEYOND FIXED DEFICIT MODELS OF ADHD}

Attention Deficit/Hyperactivity Disorder (ADHD) is a prevalent child and adolescent condition, which often persists into adulthood, marked by developmentally inappropriate and pervasive expressions of inattention, overactivity and impulsiveness (Banaschewski et al., in press). It is associated with functional impairments across multiple academic and social domains and is commonly accompanied by a range of externalising (e.g., conduct disorder) and internalising (e.g., anxiety) comorbid disorders (Taylor and SonugaBarke, 2008). Until recent times dominant neuropsychological theories of ADHD have focused on cognitive impairments associated with deficits in executive functions such as inhibitory control (Nigg, 2005) and working memory (Rapport et al., 2008). These are postulated to be grounded in altered fronto-striatal neural circuits modulated by catecholamine-based neuro-transmitters (Arnsten, 2009). Support for executive impairments in ADHD of the sort predicted by these models come from many studies (e.g. Willcutt et al., 2005; Bitsakou et al., 2008). However, it now seems unlikely that such deficits mediate the link between ADHD and its underlying causes (i.e., genes and environments; Nigg et al., 2005) in any straightforward way (Castellanos et al., 2006). For instance, many children with ADHD appear not to have dysfunctional executive processes, while many children without ADHD, either with others disorders or with no disorder at all, do (Willcutt et al., 2005). Even where ADHD and executive dysfunction co-present the pattern is 
rather fragmented and variable so that pervasive and severe executive deficit appears quite rare (Nigg et al., 2005). Perhaps reflecting the influence of the classical bio-medical framework (Singh, 2008) and neuropsychological models of brain insult (Loge et al., 1990), executive dysfunction in ADHD tends to be seen as a fixed, core cognitive deficit that is largely context and state independent (Sonuga-Barke, 1994). However, this is probably an inaccurate characterisation. Executive functions are themselves dynamic processes and performance on executive tasks will fluctuate from state-tostate and setting-to-setting (Nigg and Casey, 2005). In keeping with this, context has been shown to play an important role in determining the extent to which performance on executive tasks is deficient in ADHD (cf. Shiels et al., 2008; but see also Shanahan et al., 2008).

An alternative class of theoretical constructs have also been used to explain neuropsychological impairment in ADHD. These emphasize the dynamic, rather than the fixed, nature of ADHD and especially the role of contextual and state factors in determining cognitive and performance deficits (Castellanos et al., 2006). According to these theories deficits are secondary effects of failures of more deep-seated motivational or energetical systems and processes. Although such constructs have a long pedigree in the field of ADHD research, mainstream interest in them has increased in recent years as the limitations of fixed deficit models have become apparent. In this paper we focus on two of the first theoretical constructs of this type to be defined in the 
literature; Delay Aversion (DAv; Sonuga-Barke et al., 1992) and State Regulation Deficits (SRD; van der Meere and Sergeant, 1988).

Although the DAv and the SRD constructs were initially proposed, and have since evolved independently of one another, their shared focus on the context-dependent nature of ADHD begs the question of whether these are really distinct notions or rather just the same underlying concept expressed in different theoretical language. For example, are the DAv and SRD explanations of why a child with ADHD fails to withhold a response to a delayed signal (which they frequently do) really distinct from one another? On the face of it, it would seem so. From a DAv perspective this would be regarded as an attempt to escape from delay while from the SRD perspective it would represent a failure to modulate levels of arousal/activation to respond to changes in psychological/physiological state over time (SRD)? But can these explanations be operationalised precisely in terms of brain or behavioural responses and if so do they provide us with differential predictions?

Given this uncertainty it is perhaps surprising that there has been no systematic in-depth attempt to compare and contrast DAv and SRD so far in the literature. In the current paper we set out to provide such an analysis. We ask; to what extent can the DAv and SRD constructs be differentiated in valid and useful ways in that they can (i) be demonstrated to be grounded in substantially different causes and processes and; (ii) be operationalised in a way that allows different predictions about brain activity, behaviour and 
performance deficits to be made? Alternatively, do DAv and SRD simply refer to the same underlying processes and make the same predictions but describe them using different terminology, the origins of which lie in different meta-theoretical traditions? In order to answer these questions we will need to specify each construct as clearly as possible and where necessary resolve uncertainties and ambiguities. This paper is the result of a long process in which advocates of DAv and SRD were brought together to wrestle with these issues. Through a process of mutual conceptual and theoretical interinterrogation we aim to; (i) clarify and operationalise each construct through contrasting them with the other and; (ii) establish a number of agreed upon predictions associated with one and not the other construct. Once this has been achieved a set of pivotal tests can then be jointly designed and implemented to differentiate the presence of one or other pattern of deficit.

As well as emphasizing the notion of a fixed core deficit, the classic bio-medical framework has encouraged the notion that ADHD is pathophysiologically homogeneous and mediated by a unitary set of neuropsychological impairments affecting all ADHD cases. From this perspective the ultimate goal of differentiating DAv and SRD in the current paper would be to develop a test that can ultimately prove one theory right and one wrong; The question being; is ADHD the results of DAv or SRD? While there remains the possibility that this is the case recent studies suggest that such a conceptualisation may be too simplistic. Heterogeneity is an established characteristic of ADHD at the level of etiology (with different 
genes and different environments being implicated in different individuals; Zhou et al., 2008) and at the level of clinical presentation (as captured by diagnostic subtypes; Brookes et al., 2008). It now appears that heterogeneity expressed at these levels is also mirrored at a neuropsychological level: ADHD children are each affected in different ways to varying degrees by different neuropsychological impairments. It now seems unlikely that a single deficit or pattern of deficits can account for ADHD in all or even the majority of cases - be it executive dysfunction, DAv or SRD. Evidence for this comes from a number of sources. First, the effect size of case-control differences in neuropsychological or neurological deficits are never more than moderate (Cohen's $d<.7$; Willcutt et al., 2008) with ADHD accounting for only a small proportion of variation in these factors. This stands in contrast to other cases such as the role of phonological encoding problems in reading disability where case-control difference effect sizes are of an order of magnitude consistent with a more pathophysiologically homogeneous condition (Lubke et al., 2007). Furthermore, only a sub-sample of ADHD cases seem to show any one deficit to a clinically significant degree and then rarely in a sufficiently pervasive and severe form to suggest a unitary cause for all children (Nigg et al., 2005). Finally, when two distinctly different impairments are studied in the same sample different patients appear to be affected by each one. For instance, the classic paper by Solanto et al., (2001) illustrates this most clearly - DAv and inhibitory control deficits were uncorrelated but yet both were associated with ADHD: Four subgroups of children could be identified - DAv and no inhibitory deficits; inhibitory deficits and no DAv; both deficits and neither deficits (see 
also Thorell, 2007).More recently, a similar pattern was found for SRD and executive deficits (but not DAv; Wåhlstedt et al., 2009).

Recognising the likelihood of pathophysiological heterogeneity in ADHD we need to review the ultimate purpose of the current analysis. Rather than asking the question - Which of these two theories is correct? - our guiding question might more appropriately be framed as; Do the DAv and SRD constructs, in as much as they can be distinguished from one another at a conceptual level and operationalised in terms of construct-specific predictions, provide a way of partitioning the neuropsychological heterogeneity in ADHD? Are some patients affected by DAv, others affected by SRD, still others affected by both SRD and DAv and some affected by neither? Understood in this light the test of the success of the current paper is therefore whether it can lead to the development of experimental tests that allow us to identify the presence of DAv and/or SRD in a population of ADHD children. It is of course conceivable that this is not possible and that DAv and SRD overlap conceptually and empirically to such a degree that there is no justification for their continued existence as separate concepts. Alternatively, although distinguishable at a conceptual and methodological level, they may overlap empirically to a very great degree with children with DAv also having SRD. In either case the task becomes one of translating and integrating the two constructs rather than differentiating between them. We will return to the implications of neuropsychological heterogeneity in ADHD later in the paper as we discuss issues of practical implementation. 


\section{DELAY AVERSION \& STATE REGULATION DEFICITS; THEORETICAL FORMULATION AND CURRENT EVIDENCE}

\section{I: Delay Aversion}

Theoretical Formulation: The DAv construct is derived from a motivational model of ADHD. That is, it is one of a number of theories that explain ADHD in terms of fundamental alterations in the child's response to changes in motivational context, and the inevitable knock-on effect these have for reinforcement-based learning mechanisms (Sagvolden et al., 2005). There has been renewed interest in this sort of model (Castellanos et al., 2006; Johansen et al., 2009). However, a recent review failed to provide much convincing evidence for specific alterations in a wide range of different putative motivational mechanisms theorized, at one time or another, to be implicated in ADHD (e.g., over- or under-sensitivity to reward delivery; Luman et al., 2005). In contrast to this generally disappointing picture is the more consistent evidence for an effect of delay, prior to delivery of rewards, on ADHD children's responses and choices (Tripp and Alsop, 2001). This is often characterized as a preference for smaller sooner (SS) over larger later rewards (LL) (see Willcutt et al., 2008 for a meta-analysis; but see also Scheres et al., 2006 and Bidwell et al., 2007; for a counter case). The DAv hypothesis was initially developed to explain this pattern of performance (Sonuga-Barke et al., 1992). Consequently choice of SS over LL has become regarded as the signal mark of DA $v$ in ADHD. However, over the last 10 years or so the DAv hypothesis about SS over LL choices has been developed into a thoroughgoing and more comprehensive developmental 
model of ADHD (Sonuga-Barke, 2003). This model differentiates what might be classified as primary manifestations and secondary adaptations of DAv (Figure 1; Sonuga-Barke, 2005; Sonuga-Barke et al., 2008).

Primary manifestations: DAv is postulated to be grounded in alterations in the dopamine modulated reward circuits of the brain implicating regions such as orbito-frontal cortex and the ventral striatum (Sonuga-Barke, 2003; see below). It is driven by fundamental neuro-biological constitutionally-based alterations in the efficiency with which future rewards are signaled by dopamine within brain reward circuits (Cardinal et al., 2001; Forbes et al., 2009; Schott et al., 2008). This leads to what has been described as an impulsive drive for immediate reward (Marco et al., 2009). Crucially the model predicts that these processes and the effects they produce lead to the development over-time of negative affect which becomes associated with delay-related settings and events as children with impoverished delaysignaling associate delay settings with censure and failure (Sonuga-Barke, 2003). This exacerbates the primary tendency to choose SS over LL as escape and/or avoidance of delay become a powerful driver of behavior in delay-rich settings. This secondary motivational element is hypothesized to develop directly out of, and compound, the primarily neuro-biologically based effects. So for instance, this means that in choice setting where immediate and delayed rewards are both on offer, choices of small SS over LL rewards are motivated by two processes (a) unconditional primary preference for immediacy linked to constitutionally-based deficits in signaling future rewards and (b) the desire to escape or avoid the negative delay-related emotion 
acquired during development. These effects, significant in and of themselves, add to each other to create a marked preference for immediacy over delayed alternatives (Marco et al., 2009).

Secondary adaptations: DAv can also be manifest in non-choice settings through what we refer to here as secondary adaptations - in this sense DAv has a broad impact across different choice and non-choice settings - with effects being context specific (Sonuga-Barke, 1994; Sonuga-Barke, 2005; Bitsakou et al., 2009). So for instance, where there is a choice between different levels of delay associated with different activities and outcomes, and escape from delay is possible, children with ADHD choose SS over LL rewards or invest less time and effort in a task even though it would lead to poorer performance and associated outcomes (Sonuga-Barke et al., 2004). From its very earliest presentation the model has distinguished between such situations and others where delay is imposed and escape and avoidance of it is not possible (Sonuga-Barke, 1994). In these situations, which of course are extremely common in everyday life, the theory predicts that the DAv child's actions are differentially reinforced if they act to alter (specifically to speed up) the perception of the passage of time. In turn this will reduce subjective delay (Antrop et al., 2006). The timing literature suggests that time is extremely plastic and is altered, for instance, when attention is directed to or captured by non-temporal stimulation (Gautier and Droit-Volet, 2002a; 2002b; Brown, 1997), or when such stimulation is elicited from the environment by activity (Sonuga-Barke, 1994; 2003). According to the DAv model in ADHD these behaviours are reinforced by the reduction of subjective delay and are 
manifest as distractibility/inattention and in the latter case as hyperactive fidgeting (with a focus on behavior): Both of which would be predicted to increase with time on task. Inevitably there is a certain degree of incompatibility between these behaviours and many important tasks and so they will inevitably reduce the quality of performance where this is the case. Crucially, the probability that attention will be focused on non-temporal taskincompatible features will vary as a function of trial length or time on task. The hypothesis therefore predicts that DAv will be associated with performance deterioration on long and boring tasks with slow event rates (i.e. those that are delay rich). The model also predicts that ADHD children will show emotional arousal to unexpected imposition to delay.

One key prediction of this model of the broader ADHD phenotype is that measures of delay-related behavior in choice and no-choice tasks should share common variance. This was recently tested in a study (Bitsakou et al., 2009) that included a choice delay task (i.e., The Maudsley Index of Delay Aversion - MIDA), a delayed reaction time task (DRT; in which event rate was varied) and the Delay Frustration Task (DeFT) in which frustration was monitored following the unexpected imposition of delay during a task. Although the correlation between the outcomes was low, the choice of SS over LL (on the MIDA), the difference in reaction time between slow and fast event rates on the DRT and the degree of frustration of DeFT shared sufficient variance to form an underlying component that was more strongly associated with ADHD than performance on the three tasks separately (although a subsequent analysis suggested that there might actually be two 
separate delay-related factors; Bitsakou and Sonuga-Barke, submitted). There is therefore some initial support for the statistical overlap between primary manifestations and secondary adaptations of DAv, although this may be more complicated than initially thought.

Neurobiological Basis: It has been speculated that the biological basis of DAv is specifically associated with alterations within the cortio-striatal reward circuits (Sonuga-Barke, 2003). These circuits implicate ventral striatum (in particular nucleus accumbens) with mediated connections to the frontal regions (especially anterior cingulate and orbito-frontal cortex; Berridge and Kringelbach, 2008). These pathways are reciprocated via the ventral pallidum and related structures through the thalamus. This circuit is specifically implicated in the signaling of rewards, coding incentive salience and valence and regulating other behavioral processes involved in the maintenance of responding under conditions of delayed rewards (Cardinal et al., 2001; Cardinal, 2006). Indeed Gregorios-Pippas et al., (2009) recently demonstrated that the ventral striatum is highly sensitive to relatively minor temporal alterations on reward discounting tasks. The amygdala is postulated to play an important role in this system by defining the motivational significance of incentives and especially coding the aversive nature of delay (Plichta et al., 2009). Dopamine is a key neuro-modulator (Cools, 2008) and appears to play a special role in the control of delay-related responding (Roesch et al., 2007). To date the role of structural and/or functional alterations within this circuit in ADHD remain under-researched although recent studies suggest underactivation in the ventral striatum in ADHD following the signaling of future 
rewards (Scheres et al., 2007) and over-activation of the amygdala as delay to rewards increase (Plichta et al., 2009). Furthermore, our understanding of the functional neuroanatomy of delay-related activity more generally is developing with, for instance, the suggestion that different brain networks are differentially activated in relation to rewards and delay (Ballard and Knutson, 2009). These new perspectives need to be integrated into motivational models of ADHD. In keeping with the notion that executive deficits and DAv are dissociable elements within the pathophysiology of ADHD, they appear to be based within different brain circuits (Dalley et al., 2008). However, there has to date been no direct study of the independence of the neurobiological foundations of these distinctive elements within ADHD.

Psychopharmacology: To date there is little work specifically on the psychopharmacology of DAv in ADHD. However, by developing the construct in a number of ways we can make some tentative predictions with regards to the way in which stimulants such as amphetamine and methylphenidate should operate to reduce DAv. Two areas of literature are particularly valuable in this regard. Stimulant medication (e.g., methylphenidate and amphetamine) reduces symptoms of ADHD and improves academic and more general functioning (Taylor and Sonuga-Barke, 2008). These effects can be mirrored in improved performance on laboratory-based cognitive tasks (Coghill et al., 2007). The mechanism of action underpinning these effects still remain to be determined definitively. At a cellular level it seems fairly clear that stimulant drugs increase levels of extra-cellular dopamine either by increasing its release, (e.g., amphetamines; Joyce et al., 2007), or reducing 
its reuptake by blocking the dopamine or norepinephrine transporter (e.g., methylphenidate; Schiffer et al., 2006). Consistent with a motivational account of the therapeutic action of methylphenidate, Volkow et al. (2004) has shown that the effects of methylphenidate on performance on an academic task appear to be mediated by an increase in task salience and enhanced positive task valence. An extension of the DAv model allows a mechanism for these motivational effects to be specified. A two-stage hypothesis can be proposed: Stimulants increase the stimulus-related (phasic) dopamine signal for delayed rewards while at the same time reduce the aversive properties of delay by increasing the perceived rate at which time passes. Evidence from animal (Bizot et al., 2007) and human models (van Gaalen et al., 2006) suggest that stimulants reduce impulsiveness and increase preference for delayed over immediate rewards (Pietras et al., 2003) and speed up the 'internal clock' thereby reducing the perception of the passage of external time (Mattel et al., 2004). In general terms one would predict that the effects of stimulants on ADHD performance and behaviour are mediated by altered motivation, increased task related effort and the speeding up of time in passing during delay. The first study examining the extent to which methylphenidate reduces impulsive choice in ADHD using a discounting procedure has been published with positive results (Shiels et al., in press). Two other recent studies give rather mixed results in relation to related concepts (Risk Taking/Gambling DeVito et al., 2008; reflection/impulsivity - DeVito et al. 2008). 
Evidence Base: As mentioned above the choice of SS over LL rewards by children with ADHD on simple laboratory choice tasks is regarded as the signal indicator of DAv. The evidence in relation to this has recently been reviewed (Willcutt et al., 2008). ADHD cases can be satisfactorily differentiated from controls in terms of their choices of SS over LL rewards (Kuntsi et al., 2001; Antrop et al., 2006; Bitsakou et al., 2009; Marco et al., 2009; Sonuga-Barke et al., 1992; Schweitzer and Sulzer-Azaroff, 1995; Solanto et al., 2001; Hoerger and Mace, 2006; Dalen et al., 2004; Marco et al., 2009). Although the effect appears generally robust there is a degree of variability across studies with a number reporting negative results (Bidwell et al., 2007; Scheres et al., 2006; Wåhlstedt, et al., 2009). The reasons for these failures to replicate are unclear although sample differences and methodological factors are possible candidates. Despite this heterogeneity, the pooled size of this effect (Willcutt et al., 2008) compares favorably with those reported in the Willcutt et al., (2005) meta-analysis of executive function deficits in ADHD. Furthermore, the notion that the choice of SS over LL rewards in ADHD is the result of two processes (i.e., impairment in signaling of delayed rewards and acquired aversion to delay) is supported by a recent large scale study examining the effects of pre- and post-reward delay on choice for delayed rewards (Marco et al., 2009). In this study ADHD children chose SS over LL more than controls under both conditions - but this preference was greater when there was no post-reward delay and overall delay could be avoided. 
The notion that DAv in ADHD is a broad-based motivational style which also includes secondary adaptations in non-choice settings has been tested directly less often. In one study, ADHD children's preference for $L L$ increased when they were given access to additional stimulation - a finding consistent with the relationship between DAv and stimulation seeking (Antrop et al., 2006). However, the predictions of secondary adaptations are consistent with data from a range of different paradigms. For instance, ADHD children respond inappropriately to the unexpected imposition of delay (Bitsakou et al, 2006) and extinction of reward (Sagvolden et al., 1998); prematurely disengage from long and challenging tasks (Scime and Norvilitis, 2006); show more activity than controls during delay (Antrop et al., 2003); are biased towards task responses tied to immediate rewards (Tripp and Alsop, 2001); are unusually vigilant to environmental delay-related cues (Sonuga-Barke et al., 2004); prefer reward immediacy to high reward rate or task ease (Neef et al., 2005); discount future hypothetical rewards (Barkley et al., 2001; but see Scheres et al., 2006 for counter case); and are differentially affected by slow event rates/sparse schedules of reinforcement (Wiersema et al, 2006; Aase and Sagvolden, 2006).

In summary there is evidence to support the notion that ADHD children can be DAv in both the narrow sense of choosing SS over LL rewards to escape delay in choice situations and in the broader sense as in seen in the effects of time-on-task/trial length on performance in non-choice situations. As is the case with executive deficits these effects are relatively modest in size 
suggesting neuropsychological heterogeneity and that only a sub-set of ADHD children display DAv.

There are number of areas where further research is urgently required. We know little about the specificity of DAv to ADHD in terms of differentiating its effects from other psychiatric conditions. Indeed impulsive choice behaviour is implicated in a range of different psychopathologies as well as ADHD (e.g., conduct disorder and substance abuse; Bobova et al., 2009). In part the impulsive choices associated with substance abuse disorders and conduct disorders may be due to the overlap between these conditions and ADHD, or at least to a common neuro-biological vulnerability (Szobot and Bukstein, 2008). It is also possible that the quality of impulsive choices in ADHD is different from those associated with other disorders where ADHD is not present. For instance, in the theoretical formulation DAv is distinguished from a straight forward impulsive drive for immediate reward. This is especially so with regard to the secondary affective component that is postulated to drive the ADHD patient's motivation to escape delay and underpins their inattentive and hyperactive behaviour. Further work is needed to distinguish between these two components of DAv in the different disorders of impulse control. As far as ADHD sub-types are concerned it has been suggested that DAv may be associated with the hyperactive/impulsive dimension rather than the inattentive one. Inconsistent with the DAv theory, a recent study by Scheres et al. (2008) supported this notion. Much more work 
is also required to establish an understanding of the neuro-biology and pharmacology of DAv in ADHD.

\section{II: State Regulation Deficits}

Theoretical Formulation: A number of different models of ADHD deficits have been developed from a SRD perspective. These models emphasize that impairment in ADHD is highly variable as a function of context. In contrast to the DAv model, however, their primary focus is on the energetic state of the ADHD child and they have as their primary goal the explanation of statespecific and context dependent aspects of cognitive performance deficits. The development of SRD accounts of ADHD are situated within the cognitiveenergetic framework of information processing efficiency developed by Sanders $(1983,1998)$. In this model task efficiency is considered as a product of a combination of elementary cognitive stages (level 1 - stimulus encoding, memory search, binary decision and motor preparation) and their energy distribution (level 2 and 3 - see figure 2). Level 1components are seen as core structural processes that mediate between a stimulus and response. Therefore if deficits in ADHD were to be located exclusively at this level, independent of levels 2 and 3 , this would be consistent with a model that presents ADHD as the result of a fixed core cognitive deficit. Although not representing an executive function deficit themselves such stages are also inevitably implicated in performance on high level executive tasks, such as divided and focused attention, and (motor) set shifting. According to the cognitive energetic framework the functioning of these elementary stages at 
level 1 is regulated by the arousal and activation processes of the subject represented at level 2. Although at first arousal was seen as a unified construct, being the non-directional component of motivation, later accounts (Mulder, 1986; Sanders, 1983, 1998), have drawn a useful distinction between phasic arousal processes and tonic activation processes, with the former specifically affecting input processes (i.e., stimulus encoding) and the later affecting output processes (i.e., motor preparation). In this sense arousal is defined as a time-locked phasic physiological response to input, whereas activation refers to a long-lasting voluntary readiness for action (McGuinness and Pribram, 1980; Pribram and McGuinness, 1975). Effort (level 3) is necessary to meet task demands and to compensate for a sub-optimal energetic state by modulating arousal and activation levels. This means that performance can be maintained even in sub-optimal states or settings. The effort system is under control of an evaluation mechanism (with many features in common with executive control systems in other models), which scans the momentary state of the arousal and activation levels.

Using Sternberg's additive factor method (1969), Sanders showed that the processing stages at level 1, as well as the energetic levels 2 and 3 could be separately influenced by what he called 'task manipulations'. For instance, when the task duration is short and when subjects are motivated, stimulus degradation, set-size, and response compatibility manipulations affect the stimulus encoding, central processes and motor preparation stages in predictably different ways. Energetic processes are also affected by task characteristics. For instance, arousal level is influenced by stimulus repetition. 
Motor activation is influenced by stimulus uncertainty (as typically manifest by variable stimulus presentation rate) and psychostimulants. Effort in turn is affected by factors such as knowledge of results, contingent reward and timeon task. Crucially, for the current paper, the relationship between cognitive energetic processes and performance as affected by these factors is predicted to follow an inverted ' $U$ ' function. Deviation (increases or decreases) in arousal/activation from an optimal energetic state leads to performance decrements. Therefore the state regulation framework predicts that difficulties can arise due to over arousal/activation and/or under arousal/activation. The concept of optimality in this respect is discussed at length below.

Primary deficit: In a general sense the SRD model of ADHD deficits sees these as being due to failures to properly regulate energetical state (arousal/activation) when challenged to do so in sub-optimal settings or states. This general statement implies ADHD deficits could be situated at a number of different levels within the cognitive energetic framework. They could be due to deficits within the executive control system that co-ordinates effort allocation; in the effort system that responds to changing circumstances by modulating arousal or activation levels; or more specifically in either the arousal or the activation components of level 2 within the model. While generalized formulations implicating executive control and effort allocation have been considered (Sergeant, 2005) seminal work carried out by Sergeant and Van der Meere (for reviews, see van der Meere, 2002; Sergeant et al., 2003; Sergeant, 2005; van der Meere, 2005) identified the motor preparation 
stage as the focus of the deficit within the model and so, at the same time, implicated activation rather than general arousal, or other processes. The encoding, memory search, or decision stages were found to be largely intact in ADHD while there was a deficiency in the response organization- the stage associated with alterations in activation processes. This allowed the refinement of the SRD model of ADHD and the development of the more specific hypothesis that the locus of a possible state regulation deficit in ADHD relates to the response preparation-related activation processes associated with motor output/response organisation. The hallmark of this deficit is the impact of variations in required activation level induced by changes in task characteristics on task performance. Of these, event rate has been seen as a / the key probe of activation processes. In conditions of "over activation" brought about, for instance, by a very fast event rate, ADHD children are predicted to produce fast-inaccurate responding; whereas in conditions of "under activation", perhaps brought about by a very slow event rate, they will produce fast slow/inaccurate responding. Although in practice generating conditions for the former scenario has proved more difficult (see below).

Deficits intrinsic to aspects of the activation system could arise for a number of reasons and at present the SRD makes no specific predictions about these. Either the activation system itself could be less stable and more disturbed by alterations in context. Alternatively it could be less efficiently modulated by the effort systems (level 3) and so less efficient at counteracting 
the effects of under- or over-activation. In this sense despite this focusing in on response activation the SRD model may still implicate effort-related processes. This is the case despite the fact there does not seem to be a generalized dysregulation of effort that would be expected to disrupt the earlier information processing stages - which appears not to be the case. One way to understand how effort dysregulation could be specifically tied to activation deficits is to focus on the dynamic interplay between these two levels within the cognitive energetic framework. So that while the overall effort allocation mechanism is intact and functioning normally in ADHD in general, it could be applied inefficiently when called on specifically to modulate activation. Because of dysfunctional activation processes ADHD children may require greater levels of effort to regulate their cognitive energetic state during motor preparation. Over time the cost of applying this effort becomes excessive to the extent of being punitive. Consequently effort becomes applied less and less efficiently specifically under those conditions requiring the regulation of activation state during response preparation. In this sense failures of effort allocation might be predicted to exacerbate and compound the more fundamental deficits in motor activation. If this were the case ADHD deficit would rest at the interstices between the effort and the activation components of the model.

Secondary adaptation: Like the DAv model the general thrust of the SRD model is consistent with the idea that ADHD is associated with secondary adaptation to the primary deficit, although these claims have not 
previously been made explicit. Furthermore, there are similarities in the general features of such adaptations in the two models. For instance, it is predicted that patients may compensate for under-activation by seeking additional stimulation through their activity and patterns of attention (Alberts and van der Meere, 1992; Börger and van der Meere, 2000b). This could be seen as either a behavioural compensatory strategy or adaptation to their failure to regulate their state physiologically. This may lead to a preference to choose the more stimulating alternative where different options are available in an attempt to transform the current environment into one that is more desired or required. For instance, SRD could therefore lead to a preference for immediate reward over delayed reward if that increases the level of stimulation in the environment to either optimal or desired states (but see below for a detailed discussion). As in the case of DAv these effects are likely to be exacerbated under long and boring tasks and to lead to negative affect where state regulation through secondary adaptation is frustrated. Figure 3 provides an illustration of the SRD model described above.

Neurobiological Basis: The exact brain circuits underpinning SRD in ADHD are yet to be fully delineated. Although several brain structures and neurotransmitters have been argued to be associated with the different energetic mechanisms (Pribram and McGuinness, 1975; McGuinness and Pribram, 1980), few attempts have been made to investigate these directly. The core brain system associated with arousal extends from the spinal cord through the brainstem reticular formation including the hypothalamic sites. 
Amygdala and related fronto-cortical structures are involved in the control of the core brain arousal system. The dominant neurotransmitters in the arousal system are serotonin and norepinephrine (NE). Structures involved in the activation system are dorsal thalamus, basal ganglia and the forebrain corpus striatum. For activation, dopamine is the most important neurotransmitter. Theoretically, one would expect that other structures known to be involved in the control of physiological state such as anterior cingulate cortex (ACC) and locus coeruleus (LC) to also be implicated in SRDs in ADHD. Disturbed ACC functioning in ADHD is supported by a growing body of evidence. Several imaging studies (functional Magnetic Resonance Imaging (fMRI), positron emission tomography, single photon emission tomography) have found volumetric abnormalities of ACC and disturbed (typically reduced) activity in this brain region in individuals with ADHD (e.g., Bush et al. 1999; Ernst et al. 2003; Rubia et al. 1999; Schulz et al. 2004). Only one study so far has directly investigated ACC activity including a manipulation of activation through event rate changes. Using $\mathrm{fMRI}$ this study indicated that the ACC was involved in maintaining an optimal readiness to respond through effort allocation. Worse performance in a slow condition by adults with ADHD was accompanied, together with diminished activity in thalamus, by less activity in ACC (Kooistra et al, 2006). Together with dorsolateral prefrontal cortex the ACC has been argued to form a circuit that underlies compensatory effort allocation (Kumari et al., 2004; LaBerge, 1995; LaBerge et al., 1992; Portas et al., 1998). The LC system, located in the brainstem, has also been related to arousal/state regulation processes. Tonic activity seems to be associated with the 
regulation of arousal and a variety of state-dependent processes, such as sensory information processing, attention, working memory, and motor processes (Devilbiss and Waterhouse, 2004; Arnsten and Dudley, 2005). Some researchers have proposed that the pathophysiology of ADHD involves 'overdrive' of the LC, with excessive NE release leading to reduced capacity of the PFC to respond to phasic stimuli (Mefford and Potter, 1989; Pliszka et al., 1996). Little research has as yet been conducted on the role of LC in ADHD.

Psychopharmacology: The neurochemistry of SRD and DAv inevitably share many common elements. Activation levels have been differentially related to neurotransmitter systems in the brain. While the dominant neurotransmitters of the arousal system being serotonin and NE (Damasio, 1994; LeDoux, 1996), for activation this is dopamine (Robbins, 1998). In this regard it is interesting that $\mathrm{MPH}$ enhances task performance in children with ADHD (van der Meere et al., 1995) during conditions with a slow stimulus presentation rate. From an SRD perspective this is interpreted as evidence that slow event rates produce under-activation and that MPH increases the activation state, which in turn results in enhanced task performance (Sikstrom and Söderlund, 2007). We explore this issue in more detail below when we focus on the specific predictions of the two theories.

Evidence Base: In keeping with the motor activation deficit model it has repeatedly been found that RT performance in children with ADHD is highly sensitive to the rate at which stimuli are presented (see van der Meere, 
2005; Sergeant, 2005 for reviews). This statement holds with respect to a variety of tasks such as paired associate learning tests (Conte et al., 1986; Dalby et al., 1977), memory recognition tests (Chee et al., 1989), a Computerized Matching Familiar Figure Test (Sonuga-Barke, 2002), Go/NoGo tests (Börger and van der Meere, 2000a; Potgieter et al., 2000; van der Meere et al., 1995), the Conners CPT (Purvis and Tannock, 2000), the CPT-A (Leung et al., 2000), the stop task (Scheres et al., 2001), and tapping tasks (Rubia, et al., 1999). Children with ADHD tend to perform more poorly in conditions of relatively slow, compared to fast and moderate event rates (for reviews, see van der Meere, 2002; Sergeant et al., 2003; Sergeant, 2005; van der Meere, 2005). The typically slow and variable response style in ADHD, when stimuli are presented at a low rate, is a consistent finding in these studies, whereas findings are mixed with respect to errors of commission (Börger et al., 2000a; Potgieter et al., 2000; Scheres et al., 2001). The event rate effect persists under sustained attention conditions of more than 30 minutes. Children with ADHD were found to have a rapid decline in task efficiency over time with a slow presentation rate, but not with a fast presentation rate (for a review, see van der Meere, 2002). The ISI effect on RT performance diminishes as a function of age (van der Meere and Stemerdink, 1999), is independent of IQ level (van der Meere and van der Meere, 2004), and differentiates at the group level between ADHD and aggressive behaviour problems such as conduct disorder and oppositional defiant disorder (van der Meere et al., 2005). From the perspective of the 
SRD model these findings are consistent with the notion that ADHD are either easily under-activated or find it more difficult to counteract under-activation.

Fewer studies have investigated the key SRD prediction derived from the inverted ' $U$ ' function relating activation to performance - i.e. that either increases or decreases in activation from an optimal energetic state lead to performance decrements (van der Meere et al., 1995). Most studies have used only two presentation rate conditions (fast versus slow) which do not allow this aspect of the model to be tested. However, where three or more levels of event rate have been used evidence tends to support the idea that effects are more robust for under-activation rather than over-activation. In contrast to this general finding, recent findings from a study by Benikos and colleagues (2009), which presented a task at three levels of event rate, suggested a greater performance decline in children with ADHD in the fast, compared to the slow, condition. The scarce evidence for over-activation problems in ADHD could be due to the fact that mechanisms of overactivation are in fact intact or because it is more difficult to induce a state of over-activation as acknowledged by Sanders. This problem is complicated further as the optimal state will also be task/context dependent and will differ between children. Nevertheless, there is some literature that supports the inverted U predictions. van der Meere et al. (1995), studied the ability to inhibit responses of children with ADHD using a Go/No-Go task under fast $(I S I=1 \mathrm{~s})$, medium $(I S I=4 s)$, and slow $(I S I=8 s)$ event rate conditions. The ADHD group performed less well than controls in the fast and slow conditions but equally 
well in the medium condition. Also Sonuga-Barke (2002) found this pattern of results in a study of time use in children with ADHD. Three trial duration conditions were used $(5,10$, and $15 \mathrm{sec})$. Children with ADHD were found to perform less well than controls on trials of both 5 and $15 \mathrm{sec}$. durations, but not on trials of $10 \mathrm{sec}$. duration. We return to this issue below when we focus on developing specific predictions to differentiate the two constructs.

There is also evidence from psycho-physiological studies that dysregulated effort allocation processes may be specifically implicated in SRD deficits associated with motor activation, as predicted by the SRD hypothesis. For instance, Börger and van der Meere (2000b) (see also Börger et al., 1999) demonstrated that the slow and variable responses in a slow event rate condition were correlated with; (i) less heart rate deceleration before the onset of the "go" stimulus, and (ii) increased heart rate variability $(10 \mathrm{~Hz}$ component), reflecting, respectively, a poor readiness to respond and less effort allocation. Additional evidence that ADHD is associated with an inability to maintain an appropriate readiness to respond state because of insufficient effort allocation came from a series of event-related potential (ERP) studies. These studies showed that slow and inaccurate RT performance in the condition with a long ISI was accompanied by reduced parietal P3 amplitude indicating less effort allocation (Wiersema et al., 2005; Wiersema et al., 2006). The deficit was found to persist into adulthood (Wiersema, et al., 2006). The Kooistra et al, (2006) fMRI study mentioned above indicated that the anterior cingulate and the thalamus are involved in maintaining a state of optimal 
readiness to respond through effort allocation; during a slow event rate, these areas were less active in adults who had been diagnosed with ADHD as children.

Summary: The SRD is consistent with much of the data on the context dependent and dynamic nature of ADHD performance. Nevertheless, fundamental questions about the model remain. For instance, it is unclear how deficient motor readiness processes interact with effort allocation. It also remains unclear the extent to which SRD follows the standard 'inverted $U$ function' typically regarded as relating energetic elements to performance and the extent to which lack of empirical evidence for over-activation is due to methodological problems rather than a true absence of the effect?

\section{CAN DELAY AVERSION AND STATE REGULATION DEFICIT PROCESSES BE DIFFERENTIATED ON THE BASIS OF THEIR COMMONLY ACCEPTED SIGNAL PREDICTIONS?}

As can be seen from the above descriptions the SRD and DAv theoretical constructs share many features in common and often make similar predictions. In general terms they both highlight the dynamic and context dependent nature of task performance - i.e. that ADHD children will perform poorly under some conditions while under others they may be indistinguishable from children without the disorder. In this section we will take what have become seen as the hallmark manifestation of each construct (preference for SS over LL for DAv and poor performance under slow event rates for SRD) and; (i) explore whether they are specifically related to one 
construct and not the other and (ii) discuss ways in which more distinctive and specific predictions can be developed. First we look at event rate effects on ADHD task performance which is seen by many as the hallmark of SRD and see to what extent it actually does differentiate SRD from DAv models. In a similar way we will then take the preference for SS over LL, which is typically regarded as a hallmark of DAv, and ask could it be explained by the SRD model.

\section{Event rate effects: Can they differentiate SRD from DAv processes in ADHD?}

That changes in event rate during tasks will have a profound impact on the performance is regarded as a hallmark of SRD in ADHD. This is argued to be due to ADHD children's inability to manage non-optimal information processing conditions (i.e. remain in an optimal state) in settings that lead to under- or over-activation. However, from what has been described above event rate effects may also reflect DAv. In this case these are linked to a secondary adaptation whereby ADHD children's activity and inattention reduces the passage of time on tasks and this effect increases as trial length increases or event rate reduces. At first sight, therefore, DAv and SRD seem to make somewhat similar predictions. However, here we propose that the two theories can be differentiated, in terms of two features of the SRD not shared by DAv.

First, that SRD model predicts that the impact of activation level on performance takes the form of an inverted ' $U$ ' function whereby either 
increases or decreases in activation from an optimal energetic state is predicted to lead to performance decrements, if not efficiently managed. The prediction therefore would be that ADHD performance would follow a nonlinear function vis-a-vis event rate. There should be deficiencies under both fast and slow event rate settings while on intermediate event rate, where these map on to optimal levels of activation, no such effects would be found. In contrast, the DAv model predicts linear-like effects whereby performance deficits are expected on delayed compared with a non-delayed condition; the prediction being no difference under high event rate conditions, some differences emerging on intermediate trials and the largest effects on slow event rate conditions. While these distinctions are clear, testing them raises a number of methodological problems of a rather generic nature. This is because it is difficult to define precisely the event rate conditions required for over-activation, under-activation and optimal activation levels for any specific individual or on any particular task. It has certainly been difficult in the past to demonstrate impoverished performance under fast event conditions experimentally. We talk in absolute terms of slow, moderate and fast event rates. But these terms are in themselves problematic. By fast and slow in this context we mean fast and slow relative to what is optimal. Therefore what is fast and slow will vary as a function of task and individual characteristics. An event may seem fast on a complex task and relatively slow on a simple undemanding task. Given this, what is required is careful piloting of different event rates to ensure that on any particular task states of over and underactivation are induced. More generally much more needs to be known about 
the effect of task characteristics in defining optimal event rates. But even in the same situation different event rates will be optimal for different individuals. What is a fast event rate for some may appear slow to others. To address this future studies could employ adjusting procedures whereby event rate is altered in response to performance or preference in order to identify optimal and sub-optimal states.

Second, in SDR both event rate and MPH are regarded as important regulators of activation state and these factors are predicted to act additively to change the cognitive energetic dynamics of the task and therefore alter performance. For instance, if a person is operating at an optimal activation level in terms of event rate (perhaps as determined by the adjusting procedure described above), then MPH administration should lead to overactivation and deterioration of performance, if on the other hand, a person is operating under stimulus deprived conditions and is under-activated then adding stimulant medication will improve performance. In contrast, in the DAv hypothesis MPH is predicted to operate by increasing the salience of events and/or improving their valence by changing time perception and/or enhancing the signalling of future events and rewards. In this sense the effects of stimulant medication will be either beneficial or neutral under all conditions. The SRD model predicts that they may be detrimental if they lead to overactivation.

A third possible difference is that from an SRD perspective, the effects of $\mathrm{MPH}$ on event rate effects might operate directly on motor preparation via the 
activation component of information processing and not by increasing motivation (or as a consequence the allocation of additional effort in general). In the DAv account, as in other motivational accounts, the effects operate to change the motivational context and increase the application of effort to task which then leads to improved performance. However, given the current uncertainty over the relationship between activation and effort in SRD as described above such predictions are currently not clear cut. It may be that while the primary effects of stimulants might be on the activation processes there may be secondary effects in terms of motivation and effort. This would make the processes mediating MPH effects difficult to disentangle. However, it will be interesting in future to further explore these factors using psychophysiological methods. For example, a decrease in variability of heart rate (HRV) and an increase in the event-related potential (ERP) parietal P3 amplitude have both been found to index effort allocation (see Mulder, 1986 for overview of effort indices), while heart rate deceleration before the onset of Go signals and ERP activity such as contingent negative variation (CNV) and lateralized readiness potential (LRP) have been linked to motor activation/preparation (Jennings, 1992; Leuthold et al., 2004). ERPs may be especially informative in this regard as they may provide valuable temporal information with respect to the various stages of cognitive processing.

These differentiating features of the DAv and SRD models lead to some quite specific predictions relating to the effects of event rate and stimulant medication. In terms of task performance the SRD model will predict 
an interaction between ADHD and event rate. ADHD children would perform poorly under both slower- and faster-than-optimal event rates relative to controls. The prediction made with regard to absolute event rate will vary as a function of the optimal setting for the particular task. The DAv theory would also predict an interaction but this would take a linear-like form, with performance increasingly deteriorating as event rate increases - being best under high and worst under low rates. The two theories predict that these effects will be moderated in different ways by stimulant medication. The SRD model predicts that on the slow event rate trials, performance will deteriorate when children are off, compared to on medication, as medication will normalise the reduced activation associated with this setting. However, on fast (or optimal) event rates performance will deteriorate as a function of MPH exposure as the optimal levels of activation are exceeded and a state overactivation prevails. For DAv, as with the SRD model performance is expected to be worse 'off' medication under slow event rates. However, in contrast to the SRD model the DAv theory predicts that medication would have either a neutral or a positive effect on performance in the fast rate condition.

\section{Choice of small immediate over large delayed rewards: Can they differentiate Delay Aversion and State Regulation Deficit accounts?}

The fact that ADHD children choose SS over LL more than controls has traditionally been regarded as the hallmark of DAv. In keeping with the DAv theory this effect seems highly context dependent. It is especially pronounced where choice of the SS allows the child to reduce trial/session length (Marco 
et al., 2009) and seems to be normalised where patients are allowed access to additional stimulation during delay (Antrop et al., 2006). Can these behavioural patterns be explained in terms of SRD? The SRD concept is not normally cast in terms of choice between different settings or options but rather in terms of response to imposed task parameters. However, as the SRD concept is developed in this paper the choice of SS over LL may be understood in terms of secondary adaptation: ADHD children seek to regulate their energetic state and optimise their activation level by choosing one environment over another. They do this by modifying their environment (i.e., choosing one alternative over another) to establish an event rate that is optimal for them in terms of activation level. SS is related to higher event rate and $L L$ to lower. This raises the possibility that ADHD-related patterns of choice between SS and LL on choice delay tasks may be explicable in terms of SRD as well as DAv in as much as ADHD children choose a delay setting that is optimal for them.

However, there are a number of caveats relating to the concept of optimality as it relates differentially to performance on cognitive tasks on the one hand and choice tasks on the other. When we talk about an optimal event rate in the context of the SRD we are typically talking about the event rate that is required for or associated with optimal performance on a task - e.g., more accurate or faster less variable responding. The situation is rather different with choice-related delay (Solanto et al., 2001) where there may be no task embedded in the delay period and all that is required is to choice a reward and wait for it. In such a situation we need to extend the concept to 
encompass not only the event rate/delay required for optimal performance but also that which is desired or preferred - we might think of this as being the event rate/delay that the participants feel most "comfortable" with. Clearly this is a major extension of the way that optimality would typically be conceptualised in the SRD model. It is conceivable that the desired state mirrors the required for optimal performance in as much as individuals can become conditioned to find states of under- or over-activation in which performance that is non-optimal might be more aversive. If this were the case then, consistent with the notion that activation-performance relations follow an inverted $U$ function, SRD would be predicted to be associated with preference for SS that varies as a function of delay prior to its delivery- that very short delays before the SS would be less attractive than intermediate delays and immediate rewards, as these, like fast event rates, may be a associated with a state of over activation. In other words the intermediate delays may therefore be associated with the optimal state. One might also predict that these effects would be more strongly related to delay level (event rate) than reward magnitude per se. The issue of what constitutes a short, intermediate and long delay mirrors that raised previously with regard to event rate. This will vary from task-to-task and person-to-person and needs to be addressed with careful piloting and methodological innovation. On the other hand, it may turn out not to be appropriate to extend the concept of optimality from the required to desired state and remain within the SRD framework. The factors that determine what is preferred in a simple choice delay task such as that used by Solanto et al., (2001) may be unrelated to state regulation factors. In this 
case, in contrast to DAv, the SRD model would make no specific predictions about preference for SS over LL. Clearly these are empirical questions and research is required to disentangle these different notions of optimality as they relate to event rate/delay and ADHD.

As we point out above preference for SS in ADHD is modulated by levels of environmental stimulation during delay (Antrop et al., 2006). Do both SRD and DAv predict this? The DAv account see accessing stimulation during delay as a means of altering the perception and aversiveness of the passage of time during delay. This effect can also be seen as consistent with the SRD model at least if we assume that the concept of optimality can be extended from required to desired event rate/delay as described above. From this point of view accessing stimulation during delay can present a secondary adjustment of activation levels to optimise the stimulus setting. However, the two concepts will differ in terms of the extent to which different types of stimulation should alter performance. For the DAv model the stimulation would be most influential in reducing preference for SS if it is non-temporal in nature (i.e., de-emphasized time in passing) while temporal stimulation should make time drag and increase preference for SS. The SRD model makes no such predictions as the quality of the stimulation should be less important than the quantity. The SRD model would predict that neutral or white noise, would improve performance in states of under-activation as it would act to increase basal activation levels directly. Such noise is unlikely to affect the perception of the passage of time (although this too is an empirical question) and so the DAv would make no specific predictions with regard to white noise during 
delay. Söderlund et al., (2007) recently studied the effects of white noise on performance and found a small but significant degree of facilitation amongst ADHD children. These effects need to be extended to simple choice tasks and replicated in larger samples. Their mediating mechanisms also need to be investigated.

\section{ISSUES OF IMPLEMENTATION AND APPLICATION}

SDR and DAv share many features in common and both constructs can account for many aspects of the extant literature. However we have established that each has specific elements that predict different effects which can be disentangled using experimental techniques. Such differential predictions relate in particular to the way event rate affects performance, the significance of long versus short delay intervals prior to rewards, the quality of stimulation required to alter performance (temporal vs. non-temporal) and the impact of stimulant medication.

The analysis set out in this paper could be seen as setting up a headto-head test of the veracity of two models of ADHD to answer the question: Is ADHD a DAv or a SRD disorder? To develop tests to answer this question would in principle be fairly straightforward. However, in the introduction to the paper we introduced the concept of, and described some of the growing evidence for, neuropsychological heterogeneity in ADHD. This complicates the situation considerably. It seems increasingly unlikely that ADHD can be explained with reference to any one set of underlying psychopathological alterations, be they executive dysfunction, DAv or SRD. ADHD in different 
individuals is probably marked by different patterns of neuropsychological impairment.

Neuropsychological heterogeneity significantly alters the way we conceptualise, theorise and measure neuropsychological dysfunction in ADHD. The potential scale of such a change and the challenge it presents needs to be acknowledged. First, it shifts our research goal from just identifying differences between ADHD and non-ADHD groups in performance to partitioning heterogeneity within the group of ADHD patients as a whole. In this latter endeavour the ability to effectively characterise the pattern of deficit found at the individual patient and differentiate it from those seen in other patients becomes paramount. In this paper we have argued that we can differentiate SRD and DAv in terms of concepts and predictions and that we are able to test these predictions in terms of differences between ADHD and control children at the group level. However, it remains to be seen whether we can develop tests that are sufficiently sensitive and powerful to identify which individual ADHD children have DAv and which have SRD? First we need to develop tests that can reliably probe for different deficits (some of which have been proposed in this paper). While identifying whether an individual has a deficit which is static and unchanging in its expression represents a challenge, the task is complicated significantly in the case of $D A v$ and SRD by a number of further factors. First, is the dynamic nature of these processes and effects and the way that they vary from time-to-time and setting to setting. This means that testing may need to be carried out over 
longer intervals and on more occasions to produce valid and reliable results. Second, is that differentiating these different elements relies on dissecting some rather subtle distinctions - with the two different underlying patterns of impairment expressing themselves in very similar ways in many settings. In order to address these challenges we need to work towards the development of tests that can effectively disentangle such dynamic and context specific processes by employing experimental procedures when contextual factors are manipulated systematically. From such experimental tests one can then potentially derive indexes of the impact of different environmental manipulations. We need to identify what will be the most reliable and robust predictors of a particular component. So for instance, a test of the effect of event rate on performance may be able to characterise those patients with a 'U shaped' (SRD) from a linear function (DAv). However, this will inevitably be a highly involved process given the complexities of establishing optimal conditions with regard to fast, moderate and slow event rates. It may therefore be that the preference for SS over LL is more useful differentiator between SRD and DAv types. Establishing the reliability, validity and the diagnostic properties, of such tests is of course essential but not straight forward. The challenge is complicated even further by the possibility that a particular child may be affected by both DAv and SRD, in the same way that some children appear to be affected both by DAv and executive dysfunction (Solanto et al., 2001). Furthermore, it is currently unclear how the co-occurrence of DAv and SRD would manifest itself in terms of performance on a event rate or choice delay tests. 
The development of tests that could classify ADHD children as having DAv and/or SRD is clearly potentially an important goal and we need to think clearly about a research strategy to follow. In order to achieve this we require a different research strategy to those currently employed. Crucially, we need to combine the sorts experimental tasks described above where contextual factors are manipulated to examine the effects of event rate or prereward delay with multivariate approaches to modelling individual difference within the ADHD group. This would allow us to identify sub-clusters of patients with large samples of ADHD with different neuropsychological deficits and define their boundaries and degree of overlap between them. These clusters can then be explored in relation to external criteria (etiological factors, prognosis and treatment response) and signal neuropsychological markers can then be identified and validated.

\section{IN SUMMARY}

In this paper we have highlighted the context dependent nature of ADHD performance and behaviour and described two different theoretical constructs; SRD and DAv that have been developed to account for this. While these two notions share some features we established their conceptual and theoretical distinctiveness and identified a number of differential predictions especially in relation to the effects of event, choice delay, environmental stimulation and stimulant medication. While in principle it is possible to distinguish these two patterns of impairment in practice the existence of neuropsychological heterogeneity complicates this task considerably. Future research needs to 
combine experimental and large scale multivariate studies to characterise and validate sub-clusters of patients with DAv and SRD and differentiate them from ADHD children with other deficits. 


\section{References}

Aase, H., \& Sagvolden, T. (2006). Infrequent, but not frequent, reinforcers produce more variable responding and deficient sustained attention in young children with attention-deficit/hyperactivity disorder (ADHD). Journal of Child Psychology and Psychiatry, 47, 457-471.

Alberts, E. \& Van der Meere, J.J. (1992). Behavioural observations of hyperactive children during sustained attention. Journal of Child Psychology and Psychiatry, 33, 1355-1364.

Antrop, I., Roeyers, H., Van Oost, P., \& Buysse, A. (2003). Stimulation seeking and hyperactivity in children with ADHD. Journal of Child Psychology \& Psychiatry, 41, 225-231.

Antrop, I., Stock, P., Verté, S., Wiersema, J. R., Baeyens, D., \& Roeyers, H. (2006). ADHD and delay aversion: the influence of non-temporal stimulation on choice for delayed rewards. Journal of Child Psychology and Psychiatry, 47(11), 1152-1158.

Arnsten, A. (2009). The Emerging Neurobiology of Attention Deficit Hyperactivity Disorder: The Key Role of the Prefrontal Association Cortex. Journal of Pediatrics 154(5), S22-S31.

Arnsten, A. F., \& Dudley, A. G. (2005). Methylphenidate improves prefrontal cortical cognitive function through alpha2 adrenoceptor and dopamine D1 receptor actions:relevance to therapeutic effects in Attention Deficit Hyperactivity Disorder. Behavioral Brain Functions, 1:2. 
Ballard, K., \& Knutson, B. (2009). Dissociable neural representations of future reward magnitude and delay during temporal discounting. Neurolmage, 45(1), 143-150.

Banaschewski, T., Sergeant, J. A., Sonuga-Barke, E. J. S., Coghill, D., Döpfner, M., Rohde, L., et al. (in press). Attention-Deficit Hyperactivity Disorder (ADHD) and Hyperkinetic Disorder (HKD). London: Oxford.

Barkley, R. A., Edwards, G., Laneri, M., Fletcher, K., \& Metevia, L. (2001). Executive functioning, temporal discounting, and sense of time in adolescents with attention deficit hyperactivity disorder (ADHD) and oppositional defiant disorder (ODD). Journal of Abnormal Child Psychology, 29, 541-556.

Benikos, N., \& Johnstone, S. J. (2009). Arousal-state modulation in children with AD/HD. Clinical Neurophysiology, 120(1), 30-40.

Berridge, K. C., \& Kringelbach, M. L. (2008). Affective neuroscience of pleasure: reward in humans and animals. Psychopharmacology, 199, 457-480.

Bidwell, L. C., Willcutt, E. G., DeFries, J. C., \& Pennington, B. F. (2007). Testing for Neuropsychological Endophenotypes in Siblings Discordant to Attention Deficit/Hyperactivity Disorder. Biological Psychiatry, 62, 991998.

Bitsakou P., \& Sonuga-Barke, E. J. S. (submitted). Dissecting neuropsychological heterogeneity in ADHD: Are inhibitory dysfunction, temporal processing deficits, and delay aversion dissociable and familial? Biological Psychiatry. 
Bitsakou, P., Antrop, I., Wiersema, R., Sonuga-Barke, E. J. S. (2006).

Probing the Limits of Delay Intolerance: Preliminary Young Adult Data from the Delay Frustration Task (DeFT). Journal of Neuroscience Methods, 151(1), 38-44. .

Bitsakou, P., Psychogiou, L., Thompson, M., \& Sonuga-Barke, E. (2009).

Delay Aversion in Attention Deficit/Hyperactivity Disorder: An empirical investigation of the broader phenotype. Neuropsychologia, 47, 446-456.

Bitsakou, P., Psychogiou, L., Thompson, M., \& Sonuga-Barke, E.J.S. (2008).

Inhibitory deficits in attention-deficit/hyperactivity disorder are independent of basic processing efficiency and IQ. Journal of Neural Transmission, 115, 261-268.

Bizot, J. C., Chenault, N., Houze, B., Herpin, A., David, S., Pothion, S., \& Trovero, F. (2007). Methylphenidate reduces impulsive behaviour in juvenile Wistar rats, but not in adult Wistar, SHR and WKY rats. Psychopharmacology, 193, 215-223.

Bobova, L., Finn, P. R., Rickert, M. E., \& Lucas, J. (2009). Disinhibitory psychopathology and delay discounting in alcohol dependence: Personality and cognitive correlates. Experimental and Clinical Psychopharmacology, 17(1), 51-61.

Börger, N. A., \& van der Meere, J.J. (2000a). Motor control and state regulation in children with ADHD: A cardiac response study. Special issue: Biological Psychology, 51, 247-267. 
Börger, N., \& van der Meere, J. (2000b). Visual behaviour of ADHD children during an attention test: An almost forgotten variable. Journal of Child Psychology and Psychiatry and Allied Disciplines, 41(4), 525-523.

Börger, N., van der Meere, J., Ronner, A., Alberts, E., Geuze, R. \& Bogte, H., (1999). Heart rate variability and sustained attention in ADHD children. Journal of Abnormal Child Psychology, 27(1), 25-33.

Brookes K. J., Xu, X. Anney, R., Franke, B., Zhou, K., Chen, W., et al. (2008). Association of ADHD with genetic variants in the 5prime-region of the dopamine transporter gene: Evidence for allelic heterogeneity. American Journal of Medical Genetics Part B: Neuropsychiatric Genetics, 147B(8), 1519-1523.

Brown, S. W. (1997). Attentional resources in timing: interference effects in concurrentn temporal and nontemporal working memory tasks.

Perception \& Psychophysics, 59(7), 1118-1140.

Bush, G., Frazier, J. A., Rauch, S.L., et al. (1999). Anterior cingulate cortex dysfunction in attentiondeficit-hyperactivity disorder revelaed by fMRI and the counting stroop. Biological Psychiatry, 45, 1542-1552

Cardinal, R. N. (2006). Neural systems implicated in delayed and probabilistic reinforcement. Neural Networks, 19(8), 1277-1301.

Cardinal, R. N., Pennicott, D. R., Sugathapala, C. L., Robbins, T. W., \& Everitt, B. J. (2001). Impulsive choice induced in rats by lesions of the nucleus accumbens core. Science, 292, 2499-2501. 
Castellanos, F. X., Sonuga-Barke, E. J., Milham, M. P., \& Tannock, R. (2006). Characterizing cognition in ADHD: beyond executive dysfunction. Trends in Cognitive Sciences, 10, 117-123.

Chee, P., Logan, G. D., Schachar, R. J., Lindsay, P.H., \& Wachsmuth, R. (1989). Effects of event rate and display time on sustained attention in hyperactive, normal, and control children. Journal of Abnormal Child Psychology, 17, 371-391.

Coghill, D. R., Rhodes, S. M., \& Matthews, K. (2007). The Neuropsychological Effects of Chronic Methylphenidate on Drug-Naive Boys with AttentionDeficit/Hyperactivity Disorder. Biological psychiatry, 62(9), 954-962.

Conte, R., Kinsbourne, M., Swanson, J. M., Zirk, H., \& Samuels, M. (1986). Presentation rate effects on paired associate learning by attention deficit disordered children. Child Development, 57, 681-688.

Cools, R. (2008). Role of dopamine in the motivational and cognitive control of behavior. Neuroscientist, 14, 381-95.

Dalby, J. T., Kinsbourne, M., Swanson, J. M., \& Sobol,M. P. (1977). Hyperactive children's underuse of learning time - correction by stimulant treatment. Child Development, 48, 1448-1453.

Dalen, L., Sonuga-Barke, E.J.S., \& Remington, R.E. (2004). Inhibitory deficits, delay aversion and preschool AD/HD. Implications for the dual pathway model. Neural Plasticity, 11, 1-11.

Dalley, J. W., Mar, A. C., Economidou, D., \& Robbins, T. W. (2008).

Neurobehavioral mechanisms of impulsivity: Fronto-striatal systems and 
functional neurochemistry. Pharmacology Biochemistry and Behavior, 90(2), 250-260.

Damasio, A. R. (1994). Decartes Error. Emotion, Reason, and the Human Brain. New York: Avon Books.

Devilbiss, D. M., \& Waterhouse, B. D. (2004). The effects of tonic locus ceruleus output on sensory-evoked responses of ventral posterior medial thalamic and barrel field cortical neurons in the awake rat. Journal of Neuroscience, 24, 10773-10785.

DeVito, E., Blackwell, A. D., Kent, L., Ersche, K. D., Clark, L., , Salmond, C. H., et al. (2008). The Effects of Methylphenidate on Decision Making in Attention-Deficit/Hyperactivity Disorder. Biological Psychiatry, 64(7), 636-639.

Ernst, M., Kimes, A. S., London, E. D., Matochik, J. A., Eldreth, D., Tata, S., Contoreggi, C., Leff, M., \& Bolla, K. (2003). Neural substrates of decision making in adults with attention deficit hyperactivity disorder. American Journal of Psychiatry, 160, 1061-1070.

Forbes, E. E., Brown, S. M., Kimak, M., Ferrell, R. E., Manuck, S. B., \& Hariri, A. R. (2009). Genetic variation in components of dopamine neurotransmission impacts ventral striatal reactivity associated with impulsivity. Molecular Psychiatry, 14(1), 60-70.

Gautier, T., \& Droit-Volet, S. (2002a). Attention and time estimation in 5- and 8-year old children: a dual-task procedure. Behavioural Processes, 58, 57-66. 
Gautier, T., \& Droit-Volet, S. (2002b). Attentional distraction and time perception in children. International Journal of Psychology, 37, 27-34.

Gregorios-Pippas, L., Tobler, P. N., \& Schultz, W. (2009). Short-Term Temporal Discounting of Reward Value in Human Ventral Striatum. Journal of Neurophysiology, 101(3), 1507-1523.

Hoerger, M.L., \& Mace, F.C. (2006). A computerized test of self-control predicts classroom behaviour. Journal of Applied Behaviour Analysis, 39, 147-159.

Jennings, J.R. (1992). Is it important that the mind is in a body? Inhibition and the heart. Psychophysiology, 29(4), 369-383.

Johansen, E., Killeen, P., Russell, V., Tripp, G., Wickens, J., Tannock, R., et al. (2009). Origins of altered reinforcement effects in ADHD. Behavioral and Brain Functions, 5(1), 7.

Joyce, B. M., Glaser, P. E. A., \& Gerhardt, G. A. (2007). Adderall® produces increased striatal dopamine release and a prolonged time course compared to amphetamine isomers. Psychopharmacology, 191, 669677.

Kooistra, L., Edwards, J., Kaplan, B., van der Meere, J., Crawford, \& Goodyear, B. (2006). Impulsivity in young adults with \& without ADHD: Evidence from functional MRI. University of Calgary, Alberta Children's Hospital Grand Rounds. 
Kumari, V., Ffytche, D.H.,Williams, C.R., \& Gray, J.A. (2004). Personality predicts brain responses to cognitive demands. Journal of Neuroscience, 24, 1036-10641.

Kuntsi, J., Oosterlaan, J., \& Stevenson, J. (2001). Psychological mechanisms in hyperactivity: I Response inhibition deficit, working memory impairment, delay aversion, or something else? Journal of Child Psychology and Psychiatry, 42, 199-210.

LaBerge, D. (1995). Attentional Processing. Harvard University Press: Cambridge, MA.

LaBerge, D., Carter, M., \& Brown, V. (1992). Positron emission tomographic measurements of pulvinar activity during an attention task. Journal of Neuroscience, 10, 613-619.

LeDoux, J. E. (1996). The emotional brain: the mysterious underpinnings of emotional life. New York: Simon \& Schuster.

Leung, J.P., Leung, P.W.L., \& Tang, C.S.K. (2000). A vigilance study of ADHD and control children: Event rate and extra-task stimulation. Journal of Developmental and Physical Disabilities, 12, 187-201.

Leuthold H., Sommer W., \& Ulrich R. (2004) Preparing for action: Inferences from CNV and LRP. Journal of Psychophysiology, 18, 77-88.

Loge, D. V., Straton, R. D., \& Beatty, W. W. (1990). Performance of Children with ADHD on Tests Sensitive to Frontal Lobe Dysfunction. Journal of Amer Academy of Child \& Adolescent Psychiatry, 29(4), 540-545. 
Lubke, G. H., Muthan, B., Moilanen, I. K., McGough, J. J., Loo, S. K., Swanson, J. M., et al. (2007). Subtypes Versus Severity Differences in Attention-Deficit/Hyperactivity Disorder in the Northern Finnish Birth Cohort. Journal of the American Academy of Child \& Adolescent Psychiatry, 46(12), 1584-1593

Luman, M., Oosterlaan, J., \& Sergeant, J.A. (2005). The impact of reinforcement contingencies on $\mathrm{AD} / \mathrm{HD}$ : $\mathrm{A}$ review and theoretical appraisal. Clinical Psychology Review, 25, 183-213.

Marco, R., Miranda, A., Schlotz, W., Melia, A., Mulligan, A., Müller, U., et al. (2009). Delay and choice in ADHD: a test of the delay aversion hypothesis. Neuropsychology, 23(3), 367-380.

Matell, M. S., King, G. R., \& Meck, W. H. (2004). Differential modulation of clock speed by the administration of intermittent versus continuous cocaine. Behavioral Neuroscience, 118, 150-156.

McGuinness, D., \& Pribram, K. (1980). The neuropsychology of attention: Emotional and motivational controls. In M. C. Wittrock (Ed.), The brain and psychology (pp. 95-140). Academic Press: New York.

Mefford, I. N., \& Potter, W. Z. (1989). A neuroanatomical and biochemical basis for attention deficit disorder with hyperactivity in children: a defect in tonic adrenaline mediated inhibition of locus coeruleus stimulation. Medical Hypotheses, 29, 33-42.

Mulder, G. (1986). The concept and measurement of mental effort. In G.R.J. Hockey, A.W.K. Gaillard, \& M.G.H. Coles (Eds), Energetics and human 
information processing (pp. 175-198). Dordrecht, The Netherlands: Martinus Nijhoff Publishers.

Neef, N. A., Marckel, J., Ferreri, S. J., Bicard, D. F., Endo, S., Aman, M. G., Miller, K. M., Jung, S., Nist, L., \& Armstrong, N. (2005). Behavioral assessment of impulsivity: a comparison of children with and without attention deficit hyperactivity disorder. Journal of Applied Behavioural Analysis, 38(1), 23-37.

Nigg, J. T. (2005). Neuropsychological theory and findings in ADHD: The state of the field and salient challenges for the coming decade. Biological Psychiatry, 57, 1424-1435.

Nigg, J. T., \& Casey, B. J. (2005) An integrative theory of attentiondeficit/hyperactivity disorder based on the cognitive and affective neurosciences. Development and Psychopathology, 17, 785-806.

Nigg, J. T., Stavro, G., Ettenhofer, M., Hambrick, D., Miller, T., et al. (2005). Executive functions and ADHD in adults: Evidence for selective effects on ADHD symptom domains. Journal of Abnormal Psychology, 114, 706717.

Pietras, C. J., Cherek, D. R., Lane, S. D., Tcheremissine, O. V., \& Steinberg, J. L. (2003). Effects of methylphenidate on impulsive choice in adult humans. Psychopharmacology, 170, 390-398.

Plichta, M. M., Vasic, N., Wolf, R. C., Lesch, K. P., Brummer, D., Jacob, C., et al. (2009). Neural Hyporesponsiveness and Hyperresponsiveness During Immediate and Delayed Reward Processing in Adult AttentionDeficit/Hyperactivity Disorder. Biological psychiatry, 65(1), 7-14. 
Pliszka, S. R., McCracken, J. T., \& Maas, J. W. (1996). Catecholamines in attention- deficit hyperactivity disorder: Current perspectives. Journal of the American Academy of Child and Adolescent Psychiatry, 35, 264-72.

Portas, C. M., Rees, G., Howseman, A. M., Josephs, O., Turner, R., \& Frith, C. D. (1998). A Specific Role for the Thalamus in Mediating the Interaction of Attention and Arousal in Humans. The Journal of Neuroscience, 18(21), 8979-8989.

Potgieter, S., Börger, N., van der Meere, J. J., \& de Cock, P. (2000). Motor inhibition in very low birth weight children associated with ADHD. Developmental Medicine and Child Neurology, 42, 17-18.

Pribram, K. H., \& McGuinness, D. (1975). Arousal, activation, and effort in the control of attention, Psychological Review, 82, 116-149.

Purvis, K. L., \& Tannock, R. (2000). Phonological processing, not inhibitory control, differentiates ADHD and reading disability. Journal of the American Academy of Child and Adolescent Psychiatry, 39, 485-494.

Rapport, M., Alderson, R., Kofler, M., Sarver, D., Bolden, J., \& Sims, V. (2008). Working Memory Deficits in Boys with Attentiondeficit/Hyperactivity Disorder (ADHD): The Contribution of Central Executive and Subsystem Processes. Journal of Abnormal Child Psychology, 36(6), 825-837.

Robbins, T. W. (1998). Arousal and attention: Psychopharmacological and neuropsychological studies in experimental animals. In R. Parasuraman (Ed.), The attentive brain, (pp. 189-219). Cambridge MA: The MIT Press. 
Roesch, M. R., Calu, D. J., \& Schoenbaum, G. (2007). Dopamine neurons encode the better option in rats deciding between differently delayed or sized rewards. Nature Neuroscience, 10, 1615-1624.

Rubia, K., Taylor, A., Taylor, E., \& Sergeant, J.A. (1999). Synchronization, anticipation and consistency in motor timing of children with dimensionally defined attention deficit hyperactivity behaviour. Perceptual Motor Skill, 89, 1237-1258.

Sagvolden, T., Aase, H., Zeiner, P., \& Berger, D. (1998). Altered reinforcement mechanisms in attention-deficit/hyperactivity disorder. Behavioral Brain Research, 94, 61-71.

Sagvolden, T., Johansen, E.B., Aase, H., \& Russell, V.A. (2005). A dynamic developmental theory of attention-deficit/hyperactivity disorder (ADHD) predominantly hyperactive/impulsive and combined subtypes. Behavioural and Brain Sciences, 28, 397-419.

Sanders, A. F. (1983). Towards a model of stress and human performance. Acta Psychologica, 53, 61-97.

Sanders, A.F. (1998). Elements of human performance: Reaction processes and attention in human skill. Mahwah, $\mathrm{NJ}$ : Lawrence Erlbaum associates.

Scheres, A., Dijkstra, M., Ainslie, E., Balkan, J., Reynolds, B., Sonuga-Barke, E., \& Castellanos, F.X. (2006). Temporal and probabilistic discounting of rewards in children and adolescents: Effects of age and ADHD symptoms. Neuropsychologia, 44, 2092-2103. 
Scheres, A., Lee, A., \& Sumiya, M. (2008). Temporal reward discounting and ADHD: task and symptom specific effects. Journal of Neural Transmission, 115(2), 221-226.

Scheres, A., Milham, M. P., Knutson, B., \& Castellanos, F. X. (2007). Ventral striatal hyporesponsiveness during reward anticipation in AttentionDeficit/Hyperactivity Disorder. Biological Psychiatry, 61, 720-724.

Scheres, A., Oosterlaan, J., \& Sergeant, J.A. (2001). Response execution and inhibition in children with AD/HD and other disruptive disorders: The role of behavioural activation. Journal of Child Psychology and Psychiatry, $42,347-357$.

Schiffer, W. K., Volkow, N. D., Fowler, J. S., Alexoff, D. L., Logan, J., \& Dewey, S. L. (2006). Therapeutic doses of amphetamine or methylphenidate differentially increase synaptic and extracellular dopamine. Synapse, 59(4), 243-251.

Schott, B. H., Minuzzi, L., Krebs, R. M., Elmenhorst, D., Lang, M., Winz, O. H., et al. (2008). Mesolimbic Functional Magnetic Resonance Imaging Activations during Reward Anticipation Correlate with Reward-Related Ventral Striatal Dopamine Release. J. Neurosci., 28(52), 14311-14319.

Schulz, K. P., Fan, J., Tang. C. Y., Newcorn, J. H., Buchsbaum, M. S., Cheung, A. M., \& Halperin, J. M. (2004). Response inhibition in adolescents diagnosed with attention deficit hyperactivity disorder during childhood: an event-related fMRI study. American Journal of Psychiatry, $161,1650-1657$. 
Schweitzer, J. B. \& Sulzer-Azaroff, B. (1995). Self-control in boys with attention deficit hyperactivity disorder: effects of added stimulation and time. Journal of Child Psychology and Psychiatry, 36, 671-686.

Scime, M., \& Norvilitis, J. M. (2006). Task performance and response to frustration in children with attention deficit hyperactivity disorder. Psychology in the Schools, 43(3), 377-386.

Sergeant, J. A. (2005). Modelling ADHD: a critical appraisal of the cognitiveenergetic model. Biological Psychiatry, 57, 1248-1255.

Sergeant, J.A., Geurts, H., Huijbregts, S., Scheres, A., \& Oosterlaan, J. (2003). The top and the bottom of ADHD: A neuropsychological perspective. Neuroscience \& Biobehavioral Reviews, 27, 583-592.

Shanahan, M. A., Pennington, B. F., \& Willcutt, E. W. (2008). Do Motivational Incentives Reduce the Inhibition Deficit in ADHD? Developmental Neuropsychology, 33(2), 137 - 159.

Shiels, K., Hawk, L. W., Jr., Lysczek, C. L., Tannock, R., Pelham, W. E., Jr., Spencer, S. V., et al. (2008). The effects of incentives on visual-spatial working memory in children with attention-deficit/hyperactivity disorder. J Abnorm Child Psychol, 36(6), 903-913.

Shiels, K., Hawk, L.W., Jr., Reynolds, B., Mazzullo, R., Rhodes, J., Pelham, W.E., Jr., Waxmonsky, J.G., \& Gangloff, B.P. (in press). The Effects of Methylphenidate on Discounting of Delayed Rewards in ADHD. Experimental and Clinical Psychopharmacology. 
Sikstrom, S., \& Söderlund, G. (2007). Stimulus-dependent dopamine release in attention-deficit/hyperactivity disorder Psychological Review, 114(4), $1047-1075$.

Singh, I. (2008). SCIENCE AND SOCIETY Beyond polemics: science and ethics of ADHD. Nature Reviews Neuroscience, 9(12), 957-964.

Söderlund, G., Sikström, S., \& Smart, A. (2007). Listen to the noise: noise is beneficial for cognitive performance in ADHD. Journal of Child Psychology and Psychiatry, 48(8), 840-847.

Solanto, M. V., Abikoff, H., Sonuga-Barke, E., Schachar, R., Logan G. D., Wigal, T., Hechtman, L., Hishaw, S., \& Turkel, E. (2001). The ecological validity of delay aversion and response inhibition as measures of impulsivity in AD/HD: a supplement to the NIMH multimodal treatment study of AD/HD. Journal of Abnormal Child Psychology, 29, 215-228.

Sonuga-Barke, E. J. (1994). Annotation: On dysfunction and function in psychological theories of childhood disorder. Journal of Child Psychology and Psychiatry, 35, 801-815.

Sonuga-Barke, E. J. (2002). Psychological heterogeneity in AD/HD - a dual pathway model of behaviour and cognition. Behavioural Brain Research, 130, 29-36.

Sonuga-Barke, E. J. (2005). Causal models of Attention-Deficit/Hyperactivity Disorder: from common simple deficits to multiple developmental pathways. Biological Psychiatry, 57(11), 1231-1238. 
Sonuga-Barke, E.J.S. (2003). The dual pathway model of ADHD. An elaboration of nero-developmental characteristics. Neuroscience \& Behavioral Reviews, 27, 593-604.

Sonuga-Barke, E.J.S., De Houwer, J., De Ruiter, K., Azensten, M., \& Holland, S. (2004). ADHD and the capture of attention by briefly exposed delayrelated cues. Evidence from a conditioning paradigm. Journal of Child Psychology and Psychiatry, 45, 274-283.

Sonuga-Barke, E.J.S., Sergeant, S., Nigg, J., \& Willcutt, E. (2008). Executive dysfunction and delay aversion in ADHD: Nosological and diagnostic implications. North American Clinics in Child \& Adolescent Psychiatry, 17, 367-384.

Sonuga-Barke, E.J.S., Taylor, E., Sembi, S., \& Smith, J. (1992). Hyperactivity and delay aversion: I. The effects of delay on choice. Journal of Child Psychology and Psychiatry, 33, 387-398.

Sternberg, S. (1969). The discovery of processing stages: Extensions of Donders' method, Acta Psychologica, 30, 276-315.

Szobot, C. M., \& Bukstein, O. (2008). Attention Deficit Hyperactivity Disorder and Substance Use Disorders. Child and Adolescent Psychiatric Clinics of North America, 17(2), 309-323.

Taylor, E. A., \& Sonuga-Barke, E. J. S. (2008). Disorders of Attention and Activity. In: M. Rutter, D. Bishop, D. Pine, S. Scott, J. S. Stevenson, E. A. Taylor, A. Thapar (Eds.), Rutter's Child \& Adolescent Psychiatry (pp. 521-123) (5 ${ }^{\text {th }}$ Edition). UK: Wiley-Blackwell. 
Thorell, L. (2007). Do delay aversion and executive function deficits make distinct contributions to the functional impact of ADHD symptoms: A study of early academic skill deficits. Journal of Child Psychology and Psychiatry, 48, 1061-1070.

Tripp, G., \& Alsop, B. (2001). Sensitivity to reward delay in children with attention deficit hyperactivity disorder (ADHD). Journal of Child Psychology and Psychiatry, 42, 691-698.

Van der Meere, J., \& Sergeant, J. (1988). Controlled processing and vigilance in hyperactivity: Time will tell. Journal of Abnormal Child Psychology, 16(6), 641-655.

Van der Meere, D. J., \& van der Meere, J., (2004). Response inhibition in children with conduct disorder and borderline intellectual functioning. Child Neuropsychology, 10(3), 189-194.

Van der Meere, J. J., Gunning, B., \& Stemerdink, N. (1995). The effect of presentation rate on task performance in ADHD children with and without tics. Journal of Perceptual and Motor Skills, 81, 259-262.

Van der Meere, J. J., Marzocchi, G. M., \& De Meo, T. (2005). Response inhibition and ADHD with and without ODD screened for a community sample. Developmental Neuropsychology, 28, 459-472.

Van der Meere, J., \& Stemerdingk, N. (1999). The development of state regulation in normal children: An indirect comparison with children with ADHD. Developmental Neuropsychology, 16(2), 213-225. 
Van der Meere, J.J. (2002). The role of attention. In S. Sandberg (Ed.), Monographs on child and adolescent psychiatry. Hyperactivity disorders. Second edition (pp. 162-213). Cambridge: University press.

Van der Meere, J.J. (2005). State regulation and attention deficit hyperactivity disorder. In D. Gozal, \& D.L. Molfese (Eds), Attention deficit hyperactivity disorder: From genes to patients (pp. 162-213). Totowa, NJ: Humana Press.

Van Gaalen, M. M., van Koten, R., Schoffelmeer, A. N. M., \& Vanderschuren, L. J. M. J. (2006). Critical involvement of dopaminergic neurotransmission in impulsive decision making. Biological Psychiatry, $60,66-73$

Volkow, N. D., Wang, G. J., Fowler, J. S., Telang, F., Maynard, L., Logan, J., Gatley, S.J,, Pappas, N., Wong, C., Vaska, P., Zhu, W., \& Swanson, J. M. (2004). Evidence that methylphenidate enhances the saliency of a mathematical task by increasing dopamine in the human brain. American Journal of Psychiatry,161(7), 1173-1180.

Wåhlstedt, C., Thorell, L., \& Bohlin, G. (2009). Heterogeneity in ADHD: Neuropsychological Pathways, Comorbidity and Symptom Domains. Journal of Abnormal Child Psychology, 37(4), 551-564.

Wiersema, J. R., van der Meere, J. J., \& Roeyers, H., (2005). State regulation and response inhibition in children with ADHD and children with earlyand continuously treated phenylketonuria: An event-related potential comparison. Journal of Inherited Metabolic Disease, 28(6), 831-843. 
Wiersema, R., van der Meere, J., Roeyers, H., Van Coster, R., \& Baeyens, D. (2006). Event rate and event-related potentials in ADHD. Journal of Child Psychology and Psychiatry, 47, 560-567.

Willcutt, E. G., Doyle, A. E., Nigg, J. T., Faraone, S. V., \& Pennington, B. F. (2005). Validity of the executive function theory of attentiondeficit/hyperactivity disorder: a meta-analytic review. Biological Psychiatry, 57, 1336-1346.

Willcutt, E.G., Sonuga-Barke, E.J.S., Nigg, J.T., \& Sergeant, J.A. (2008). Recent developments in neuropsychological models of childhood psychiatric disorders. In T. Banaschewski, L.A. Rhode (Eds.), Biological Child Psychiatry. Recent Trends and Developments. Advances in Biological Psychiatry. Basel: Karger.

Zhou, K., Chen, W., Buitelaar, J., Banaschewski, T., Oades, R. D., Franke, B., et al. (2008). Genetic Heterogeneity in ADHD: DAT1 Gene Only Affects Probands Without CD. American Journal of Medical Genetics Part B: Neuropsychiatric Genetics, 147B(8), 1481-1487. 
Figure 1: A causal model of delay aversion in ADHD.

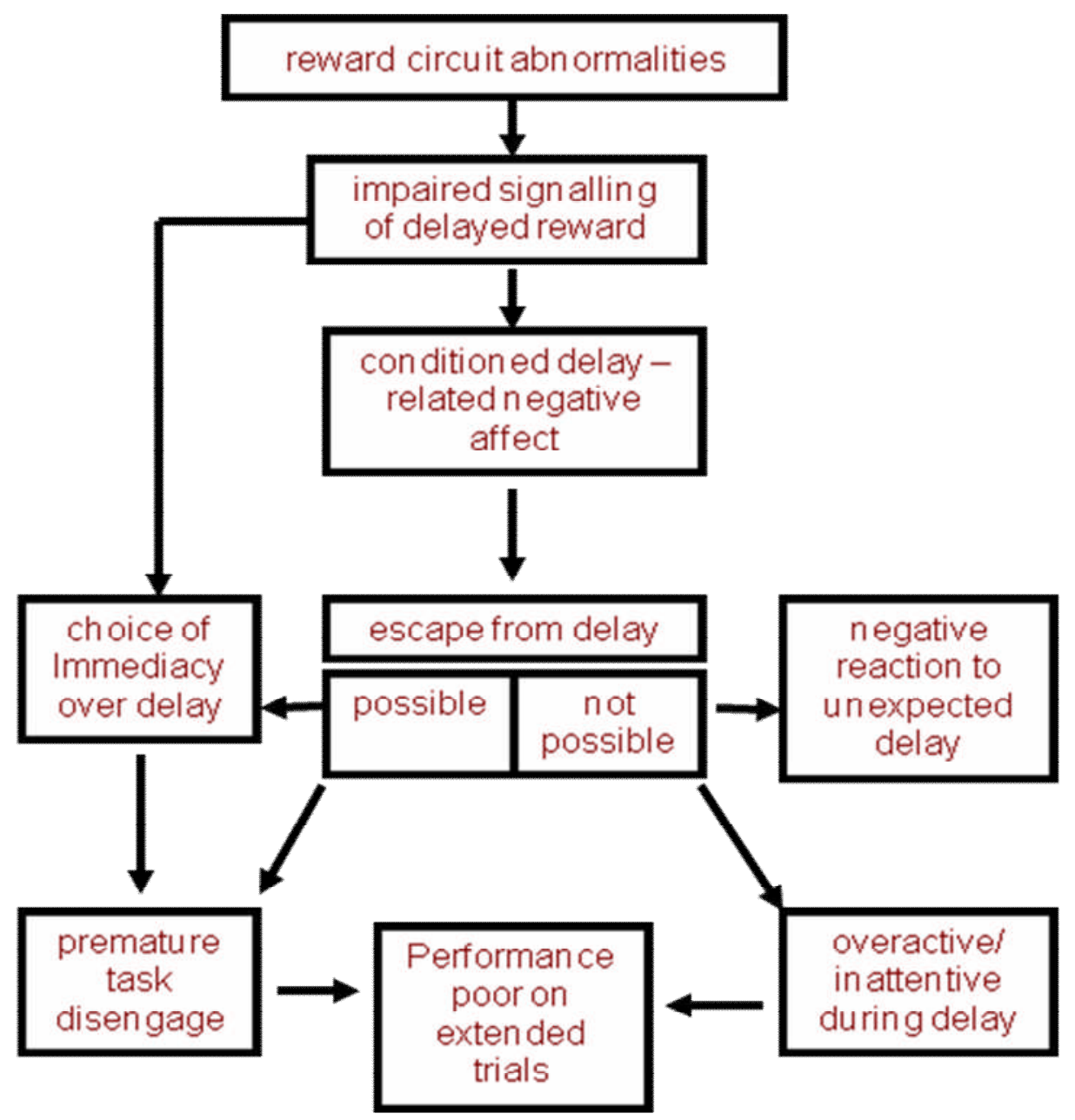


Figure 2: The cognitive energetic model of Sanders (1983).

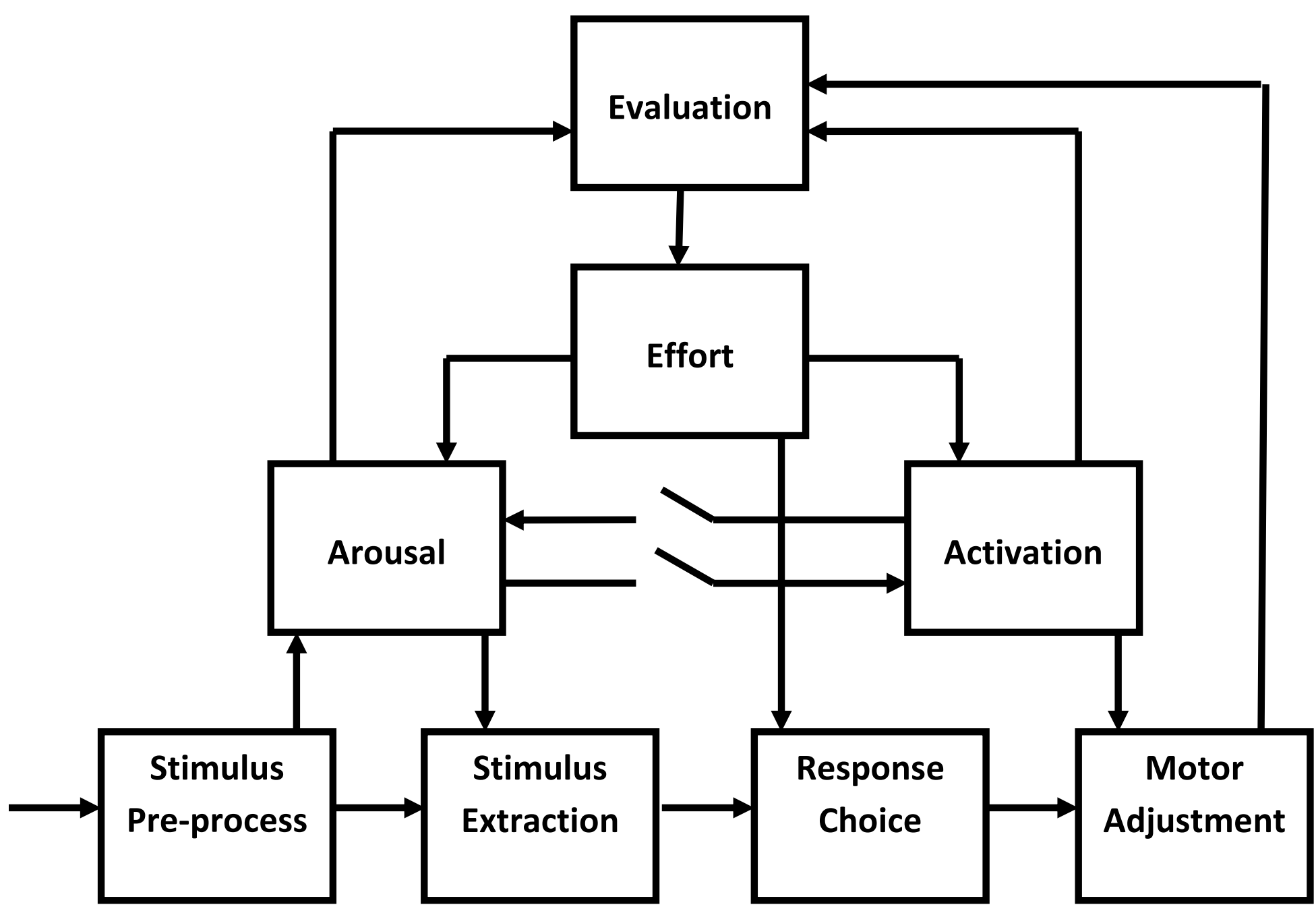


Figure 3: A possible causal model of the state regulation deficit model of ADHD.

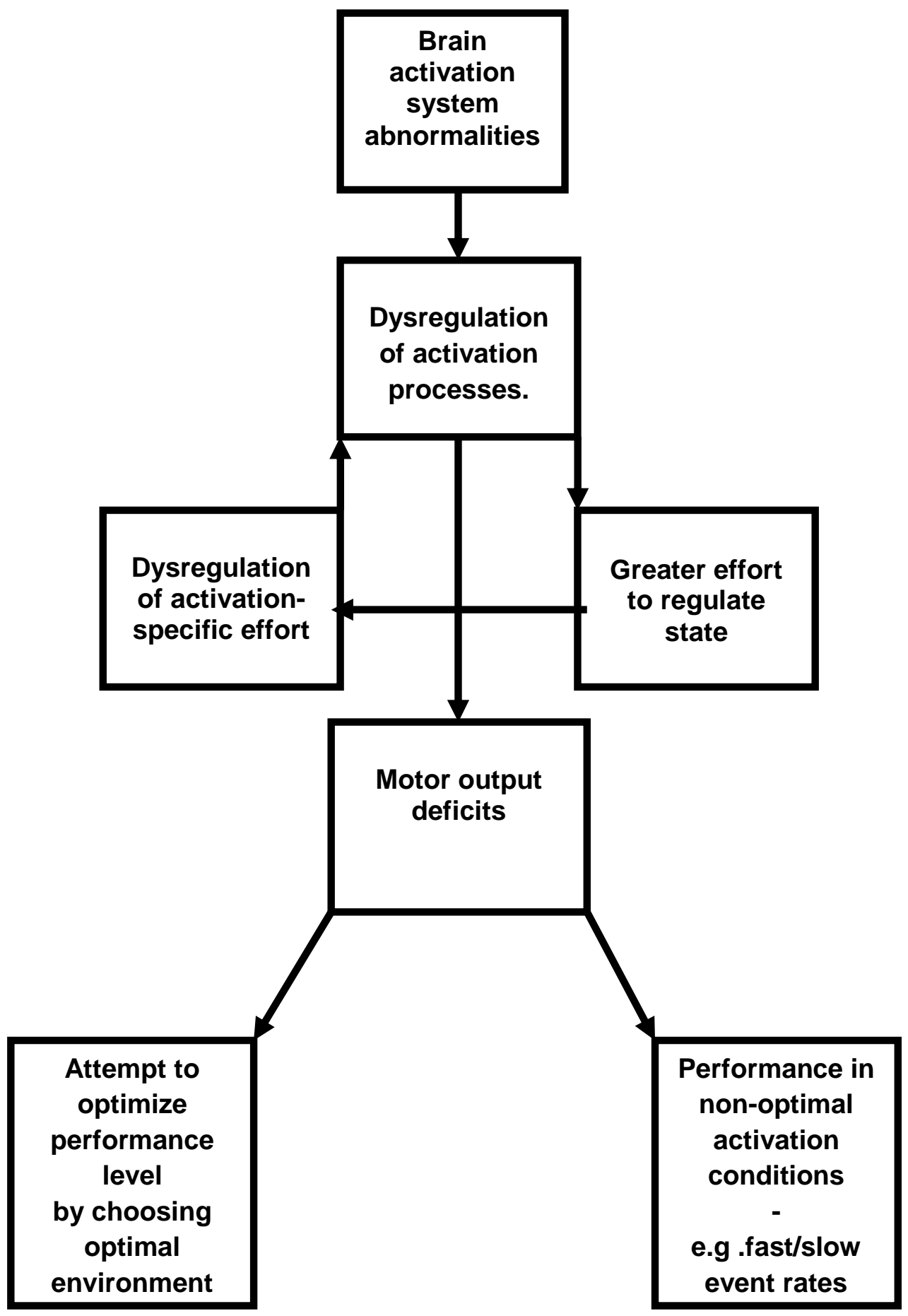

